\title{
Pulsed Laser-Assisted Helium Ion Nanomachining of Monolayer Graphene-Direct-Write Kirigami Patterns
}

\author{
Cheng Zhang ${ }^{1,2}{ }^{\oplus}$, Ondrej Dyck ${ }^{2}$, David A. Garfinkel ${ }^{1}$, Michael G. Stanford ${ }^{1}$, \\ Alex A. Belianinov ${ }^{2}$, Jason D. Fowlkes ${ }^{2}$, Stephen Jesse ${ }^{2}$ and Philip D. Rack ${ }^{1,2, * \mathbb{D}}$ \\ 1 Department of Materials Science and Engineering, University of Tennessee, Knoxville, TN 37996, USA; \\ czhang68@utk.edu (C.Z.); dgarfink@vols.utk.edu (D.A.G.); mstanfo3@gmail.com (M.G.S.) \\ 2 Center for Nanophase Materials Sciences, Oak Ridge National Laboratory, Oak Ridge, TN 37831, USA; \\ dyckoe@ornl.gov (O.D.); belianinova@ornl.gov (A.A.B.); fowlkesjd@ornl.gov (J.D.F.); sjesse@ornl.gov (S.J.) \\ * Correspondence: prack@utk.edu
}

Received: 12 September 2019; Accepted: 27 September 2019; Published: 30 September 2019

\begin{abstract}
A helium gas field ion source has been demonstrated to be capable of realizing higher milling resolution relative to liquid gallium ion sources. One drawback, however, is that the helium ion mass is prohibitively low for reasonable sputtering rates of bulk materials, requiring a dosage that may lead to significant subsurface damage. Manipulation of suspended graphene is, therefore, a logical application for $\mathrm{He}^{+}$milling. We demonstrate that competitive ion beam-induced deposition from residual carbonaceous contamination can be thermally mitigated via a pulsed laser-assisted $\mathrm{He}^{+}$milling. By optimizing pulsed laser power density, frequency, and pulse width, we reduce the carbonaceous byproducts and mill graphene gaps down to sub $10 \mathrm{~nm}$ in highly complex kiragami patterns.
\end{abstract}

Keywords: graphene; direct-write kirigami; nanopatterning; pulsed laser

\section{Introduction}

Graphene continues to attract attention as a material with intriguing physical and chemical properties and wide potential in chemical as well as biological sensors, energy conversion and storage, nanoelectronics, light-weight composite materials, and superconducting devices [1-8]. Graphene nanopatterning, also known as graphene kirigami/origami, has been intensively pursued in order to fabricate complex architectures [5,6,9-15]. Three-dimensional (3D) nanostructured graphene was investigated years ago, but a precise controlled patterning was not achieved until Blees et al. recently performed micrometer level kirigami via photolithography and plasma etching, and realized foldable 3D structures $[16,17]$. Attempts to enhance the patterning resolution have been pursued by ion milling [18-20], however, carbon contamination from the graphene transfer and patterning process, made it difficult to reproducibly push the resolution to the nanometer level. Additionally, carbon deposition in the beam interaction region due to cracking of the adsorbed hydrocarbon species or surface diffusing carbon complicates this issue [21,22]. Annealing samples in an Ar environment have been shown to reduce the carbon contamination $[18,23,24]$, however, this was insufficient to completely remove residual carbon contaminants, and thus complementary approaches are necessary to ensure the reproducibility of the high-resolution patterning.

We have explored laser-assisted focused ion and electron beam induced processing using a synchronized pulsed laser to enhance the purity of deposits [25-28], mitigate subsurface ion beam damage [29], and enhance chemically assisted etching [30,31]. In this study, a systematic study of 
laser-assisted $\mathrm{He}^{+}$milling of monolayer graphene is demonstrated. By tuning the parameters of the in situ pulsed laser beam, we mitigate competing carbon deposition and mill, as well as cut suspended graphene by the $\mathrm{He}^{+}$beam. Direct-write kirigami patterns were realized with sub $10 \mathrm{~nm}$ resolution, enabling complex nanoscale graphene fabrication. Coupled with recent developments in the area of atom-by-atom fabrication using a scanning transmission electron microscope (STEM) and conventional semiconductor manufacturing techniques, these results may suggest a pathway toward device fabrication spanning from a macro- to nanoscale and atomic scale $[32,33]$.

\section{Materials and Methods}

Commercial grade monolayer graphene samples on holey $\mathrm{Si}_{3} \mathrm{~N}_{4} \mathrm{TEM}$ membranes were purchased from Ted Pella (Redding, CA, USA). $\mathrm{He}^{+}$milling was performed with a Zeiss ORION NanoFab He/Ne ion microscope (Carl Zeiss, White Plains, NY, USA). An accelerating voltage of $25 \mathrm{kV}$ was used for all exposures. Beam currents were controlled from 1 to $3 \mathrm{pA}$. All patterns in this study were exposed with a constant pixel spacing of $0.25 \mathrm{~nm}$ and the linear dose from $1 \times 10^{4}$ to $5 \times 10^{5}$ ions/nm; the $\mathrm{He}^{+}$beam pixel dwell time is a function of total dose, current, and the number of passes. Patterns were generated using Fibics NPVE pattern generating software and hardware scan controller. The laser system used is a prototype laser delivery system developed by Waviks, Inc. (Dallas, TX, USA). It has a $100 \mu \mathrm{m}$ spot size and $915 \mathrm{~nm}$ wavelength. During the experiment the laser condition was controlled with the forward current ranging from $100 \mathrm{~mA}$ to $3 \mathrm{~A}$, the frequency ranging from 10 to $3000 \mathrm{~Hz}$, the pulse width from $1 \mu$ s to $1 \mathrm{~ms}$, and the duty cycles from $0.01 \%$ to $3 \%$. Detailed laser system information can be found in previous reports [31]. Finite element method (FEM) simulation was conducted and detailed descriptions can be found in the Supplementary Materials. STEM characterization was conducted using a Nion Ultra STEM US200 (Kirkland, WA, USA) operating at an accelerating voltage of $60 \mathrm{kV}$ with a nominal beam current of 20 pA. Graphene samples used in the STEM study were prepared in house. Chemical vapor deposition was used to grow graphene on $\mathrm{Cu}$ foil. The surface was then coated with poly(methyl methacrylate) (PMMA) and the $\mathrm{Cu}$ foil was etched away in a bath of ammonium persulfate. The PMMA/graphene stack was rinsed in deionized water and transferred to a TEM grid before removal of the PMMA with acetone. Residual hydrocarbon contaminants were removed by baking in an $\mathrm{Ar} / \mathrm{O}_{2}$ environment at $500{ }^{\circ} \mathrm{C}$ for $1.5 \mathrm{~h}$ [34].

\section{Results and Discussions}

Figure 1 illustrates typical conditions for graphene $\mathrm{He}^{+}$milling with and without in situ laser assist. Due to residual carbon contamination on the graphene samples, $\mathrm{He}^{+}$beam exposure can produce competitive carbon deposition from the cracking of the hydrocarbon species adsorbed or surface diffused into the beam interaction region. In situ pulsed laser irradiation aids to reduce the carbon concentration, which enables the high precision nanoscale milling of suspended graphene. 


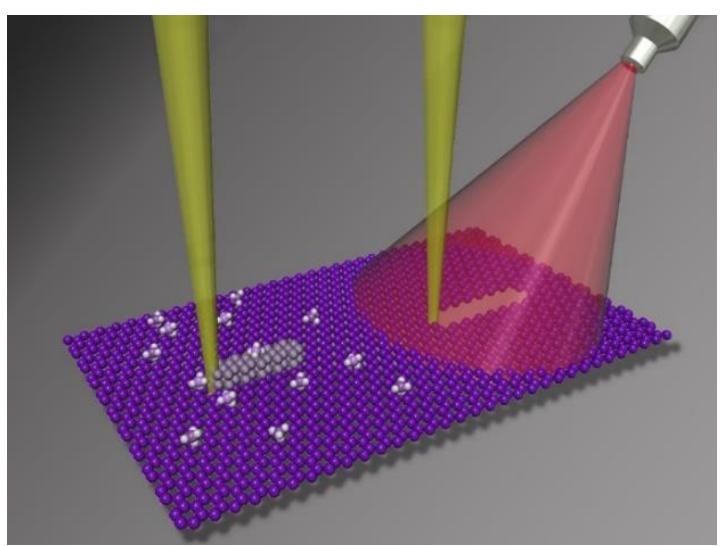

Figure 1. Schematic of $\mathrm{He}^{+}$beam exposure on a single layer graphene sample. Carbon contamination introduced during transfer process impedes a clean cut by the $\mathrm{He}^{+}$beam, and results in carbon deposition due to the cracking of residual hydrocarbon species. The carbon contamination can be reduced with the assist of an in situ pulsed laser, and thus ion milling is possible with appropriate laser conditions.

Figure 2 shows the effects of different pulsed laser conditions such as laser irradiance (power density), pulse width, and frequency when writing $400 \mathrm{~nm}$ lines with $\mathrm{He}^{+}$. In this series of tests, the $\mathrm{He}^{+}$dose was set at $2 \times 10^{5}$ ions/nm with a $0.25 \mathrm{~nm}$ pixel spacing, and the time between $\mathrm{He}^{+}$ scans in this test was at least 5 minutes, which was, as demonstrated below, adequate to re-establish the pseudo-equilibrium conditions (see Supplementary Materials, Figure $\mathrm{S} 1$ for variable $\mathrm{He}^{+}$dose). Figure 2a shows the results as a function of laser irradiance with fixed $10 \mu$ s pulse width and $100 \mathrm{~Hz}$ frequency. The bright line on top is the carbon deposition without the laser exposure. As the laser irradiance increases, less carbon deposition occurs and ion milling of the graphene proceeds at 33.5 $\mathrm{kW} / \mathrm{cm}^{2}$ (Line 3 in Figure 2a). Clean line cuts can be achieved with $\geq 38 \mathrm{~kW} / \mathrm{cm}^{2}$ laser irradiance (Line 4 to 6), where the width of milled lines is approximately $40 \mathrm{~nm}$. Figure $2 \mathrm{~b}$ and c shows the transition from deposition to milling by increasing the laser pulse width and pulse frequency, relative to a base laser condition of $29 \mathrm{~kW} / \mathrm{cm}^{2}, 10 \mu \mathrm{s}$, and $100 \mathrm{~Hz}$. The approximate threshold pulse width and frequency to induce the milling upon the base condition are $22 \mu \mathrm{s}$ and $500 \mathrm{~Hz}$, respectively. The transition is particularly sensitive to the laser irradiance and pulse width, meanwhile the deposition and milling transition evolves less with the increase of frequency. The plot of the average pulsed laser power density in Figure 2d shows that the threshold average power density (marked as dash lines) is similar for the variable laser power and pulse width series, but the frequency test has a much higher threshold. The bottom plot in Figure $2 \mathrm{~d}$, is the energy density per pulse, which helps to clarify the trends. For the frequency study, the individual peak temperature at $29 \mathrm{~kW} / \mathrm{cm}^{2}$ and $10 \mu \mathrm{s}$ is below the threshold temperature, however, at higher frequencies the refresh time is short and at $1750 \mathrm{~Hz}$ is below the room temperature equilibration time, and thus the temperature rises and eventually exceeds the threshold temperature for the carbon desorption and diffusion to occur. The detailed laser condition for all the exposures are listed in Table 1. 

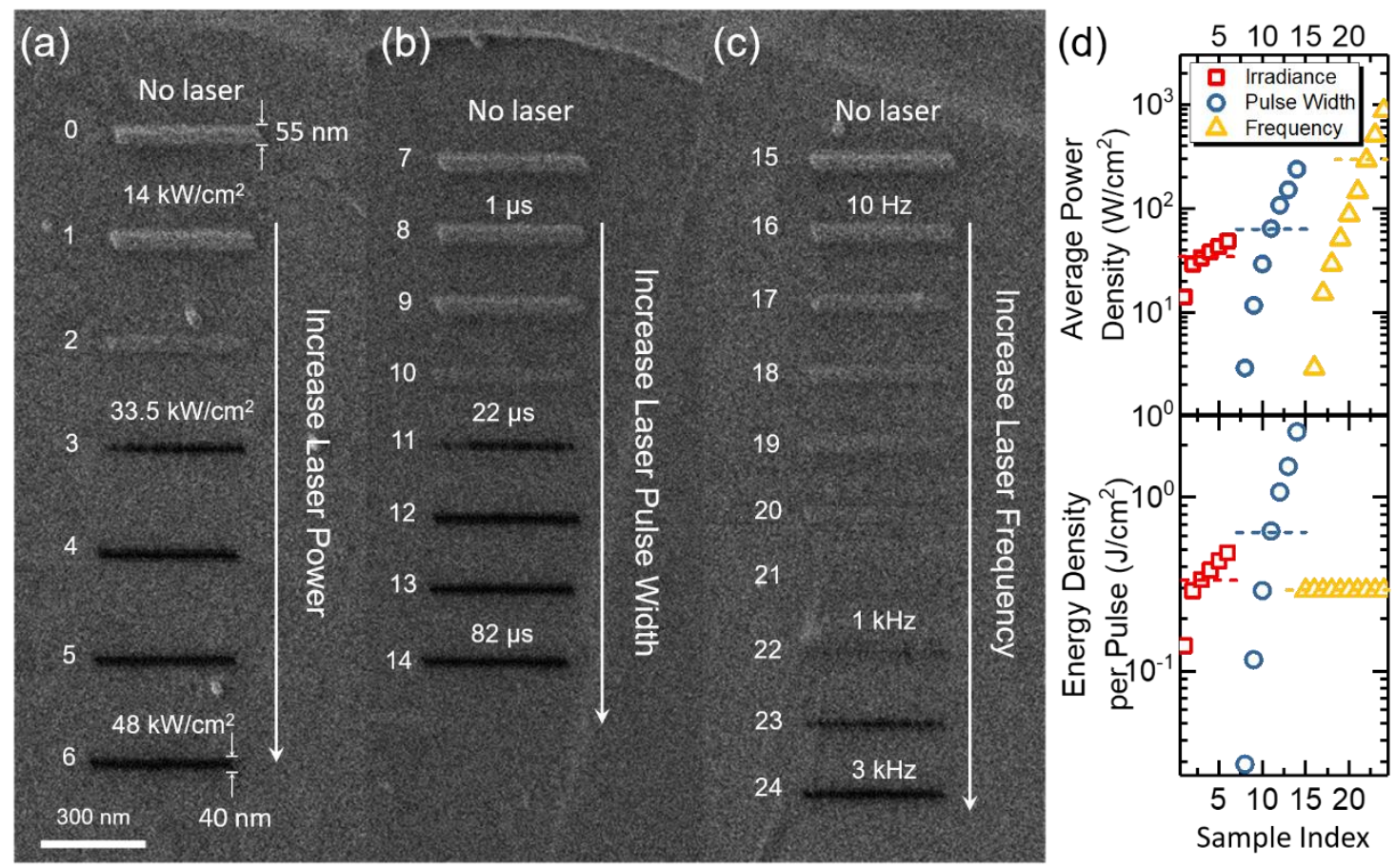

Figure 2. Tuning the laser condition via (a) irradiance, (b) pulse width, and (c) frequency to realize the transition between carbon deposition and $\mathrm{He}^{+}$milling of monolayer graphene. Constant linear $\mathrm{He}^{+}$ beam scans with the length of $400 \mathrm{~nm}$ and the dose of $2 \times 10^{5}$ ions/nm were used. The typical width of the carbon deposit and cut lines are $55 \mathrm{~nm}$ and $40 \mathrm{~nm}$, respectively. The laser pulse width and frequency were set at $10 \mu$ s and $100 \mathrm{~Hz}$, respectively, for the irradiance test shown in (a); the irradiance and frequency were set at $29 \mathrm{~kW} / \mathrm{cm}^{2}$ and $100 \mathrm{~Hz}$, respectively, for the pulse width test in (b); the irradiance and pulse width were set at $29 \mathrm{~kW} / \mathrm{cm}^{2}$ and $10 \mu \mathrm{s}$, respectively, for the frequency test in (c); and (d) plots the average laser power density and energy density per pulse. Dashed lines mark the threshold values between depositions and mills.

Table 1. Detailed laser conditions of the three tests shown in Figure 2. The base parameter was set at 29 $\mathrm{kW} / \mathrm{cm}^{2}, 10 \mu \mathrm{s}$, and $100 \mathrm{~Hz}$. Three exposure parameter series were conducted: laser power density, pulse width, and frequency.

\begin{tabular}{|c|c|c|c|c|c|c|c|c|c|c|}
\hline Line ID & 0 & 1 & 2 & 3 & 4 & 5 & 6 & & & \\
\hline $\begin{array}{l}\text { Irradiance } \\
(\mathrm{kW} / \mathrm{cm} 2)\end{array}$ & N/A & 14 & 29 & 33.5 & 38 & 43 & 48 & & & \\
\hline Line ID & 7 & 8 & 9 & 10 & 11 & 12 & 13 & 14 & & \\
\hline Pulse Width ( $\mu s)$ & N/A & 1 & 4 & 10 & 22 & 37 & 52 & 82 & & \\
\hline Line ID & 15 & 16 & 17 & 18 & 19 & 20 & 21 & 22 & 23 & 24 \\
\hline Frequency (Hz) & N/A & 10 & 52.5 & 100 & 175 & 300 & 500 & 1000 & 1750 & 3000 \\
\hline
\end{tabular}

To further understand the underlining mechanisms, FEM simulation was conducted, and the result qualitatively approximates the actual configuration used in real experiments (a detailed description of FEM can be found in Supplementary Materials). Figure 3 illustrates the expected increase in temperature with increasing power and pulse width. Furthermore, it illustrates that at $100 \mathrm{~Hz}$ frequency, the thermal decay has an adequate time $(\sim 10 \mathrm{~ms})$ to cool back to room temperature after each laser pulse. Thus, for $100 \mathrm{~Hz}$, greater than $\sim 200 \mathrm{~mJ} / \mathrm{cm}^{2}$ per pulse energy density is required to raise the temperature high enough to desorb and diffuse the carbonaceous species from the laser region of interest. Notably, at short dwell times, the peak temperature scales with both laser power density and pulse width. At a higher frequency $(>1 \mathrm{kHz})$, the thermal decay is incomplete, and the temperature does not cool to room temperature between pulses, which results in an increasing peak temperature 
as the number of pulses continues. Thus, while the initial pulse is below the temperature threshold to assist the focused ion beam milling, the steady-state peak temperature after many pulses clearly exceeds this threshold at $\sim 1750 \mathrm{~Hz}$ frequency. The FEM simulation results provide rational for the observed trends and, in particular, the higher frequency threshold, although the absolute temperatures are uncertain.

(a)

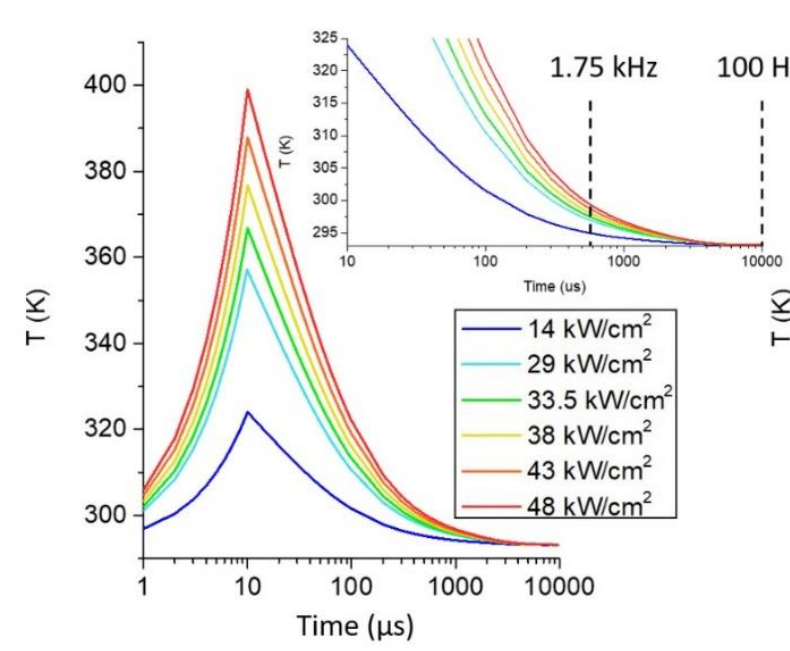

(b)

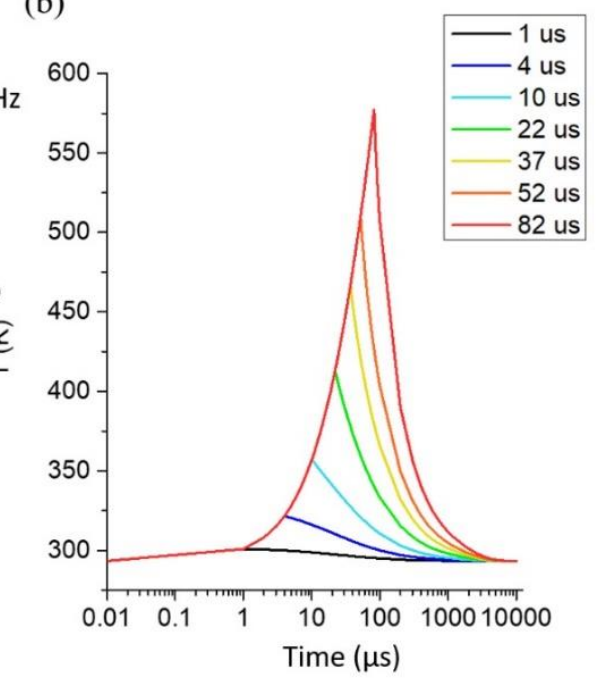

Figure 3. FEM simulation results for laser power (a) and pulse width (b) tests. The laser power profile shown in (a) is based on $10 \mu$ s pulse width and the pulse width profile shown in (b) is based on $29 \mathrm{~kW} / \mathrm{cm}^{2}$ laser power density. The laser power is calculated by $90 \%$ irradiance over a laser spot area with a diameter of $100 \mu \mathrm{m}$ (see the FEM simulation part for details). The mill happens with the power of $33.5 \mathrm{~kW} / \mathrm{cm}^{2}$ and above in (a), and with the pulse width of $22 \mu \mathrm{s}$ and above in (b), corresponding to a threshold peak temperature range of $366 \mathrm{~K}$ to approximately $411 \mathrm{~K}$.

As shown above, the pulsed laser assist can suppress the carbon deposition and enable ion milling. We attribute the suppression of carbon deposition to photothermal desorption of contaminants in the beam interaction region. Thus, for each parameter in Figure 2, a thermal threshold is reached in which the steady state carbon concentration is reduced sufficiently to allow the graphene to be milled.

Furthermore, a test was conducted to see if the photothermal treatment can permanently clean the sample for ex situ processing. Notably, the laser spot diameter is $\sim 100 \mu \mathrm{m}$, thus, a relatively large area should be impacted. Figure $4 \mathrm{a}$, b shows a comparison of the effect of a laser pretreatment on subsequent $\mathrm{He}^{+}$exposures for two different laser irradiances of 48 and $81 \mathrm{~kW} / \mathrm{cm}^{2}$, with the pulse width and frequency fixed at $10 \mu$ s and $100 \mathrm{~Hz}$, respectively. In these tests, each pattern in Figure 4a took approximately 8 to $15 \mathrm{~s}$ with a $2 \times 10^{5}$ ions/nm dose. 
(a)
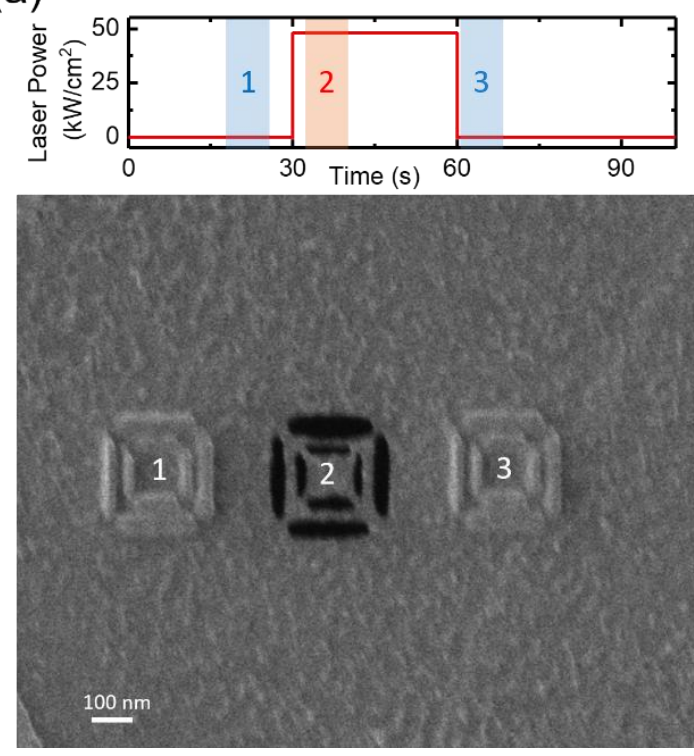

(b)
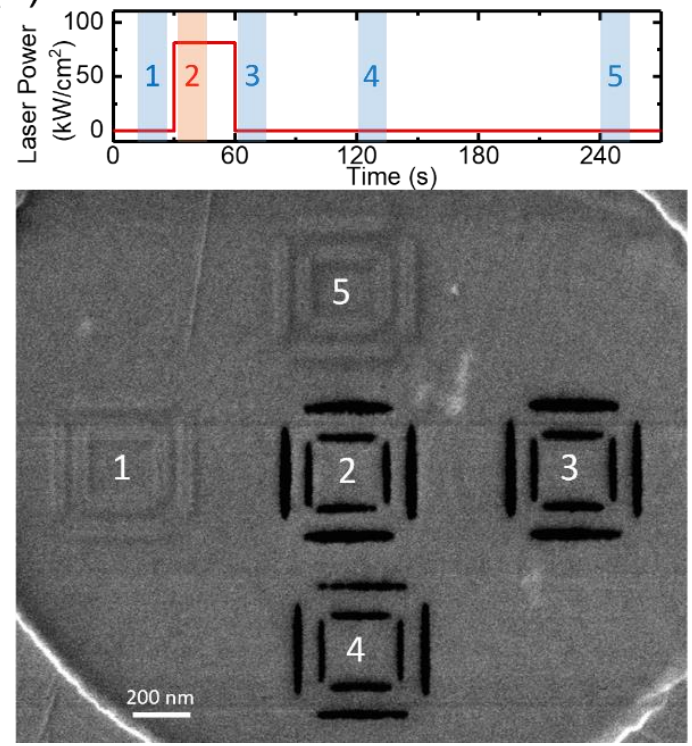

Figure 4. Effect of post-exposure time pulse laser-assisted graphene milling with low (a) and high (b) laser irradiance. Graphene milling versus carbon deposition switches immediately at $48 \mathrm{~kW} / \mathrm{cm}^{2}$ laser irradiance. By increasing the irradiance to $81 \mathrm{~kW} / \mathrm{cm}^{2}$, the residual carbon is mitigated for over $1 \mathrm{~min}$ after the laser is turned off. In this test the writing time for each pattern is 8 to 15 seconds. The in situ laser parameters were set at $10 \mu$ s pulse width and $100 \mathrm{~Hz}$ frequency and the $\mathrm{He}^{+}$dosage was at $2 \times 10^{5}$ ions/nm for all patterns.

At $48 \mathrm{~kW} / \mathrm{cm}^{2}(1 \mathrm{~A})$ laser irradiance, an immediate switch between milling and deposition is realized, as shown in Figure 4a. Pattern 1 was exposed without the laser, which led to carbon deposition and pattern 2 was exposed with the in situ laser assist and milling was observed. Then, the laser was turned off and pattern 3 was exposed immediately, and deposition was observed again. When applying the laser assist at a higher irradiance of $81 \mathrm{~kW} / \mathrm{cm}^{2}$, there was a latency time of over 1 minute in which milling was still possible. In Figure $4 b$, the first three patterns were completed following the same procedure as in Figure 4a, illustrating that the milling occurred after the laser was turned off. Pattern 4 was exposed $\sim 1$ minute after the laser was turned off-the milling still occurred, although the mill quality decreased at the edges. Finally, Pattern 5 was exposed three minutes after the laser was turned off and carbon deposition was again observed. The laser-on time for both tests was fixed at 30 seconds. Although it is not clear whether the carbon source was from the vapor phase, surface diffusion, or a combination of the two, an estimate of the surface diffusion coefficient was made by setting the diffusion distance, $x$, equal to the laser radius and solving for the diffusion coefficient (D), (i.e., $x=\sqrt{4 D t}$ ), where $t=$ time $(180 \mathrm{~s})$; a value of $\sim 3 \times 10^{-8} \mathrm{~cm}^{2} / \mathrm{s}$ was obtained which is reasonable for large molecular weight hydrocarbons [35].

Subsequent to demonstrating the enhanced milling via the laser assist, results of pattern resolution testing are demonstrated in Figure 5. The laser was set to $48 \mathrm{~kW} / \mathrm{cm}^{2}, 10 \mu$ s pulse width and $100 \mathrm{~Hz}$, and the $\mathrm{He}^{+}$linear dose was $2 \times 10^{5}$ ions/nm. Figure 5 a shows an overview of a series of patterns comparing parallel cuts, corner cuts, and vertical cuts. Figure $5 b-d$ is high magnification images indicated by the white dashed squares in Figure 5a. The highest resolution of the graphene nanobridges created by ion milling reached $10 \mathrm{~nm}$ for parallel cuts and approximately $6 \sim 7 \mathrm{~nm}$ in corner and vertical cuts. The width of the milled gaps varied from $15 \mathrm{~nm}$ to $50 \mathrm{~nm}$, which was due to the deformation of graphene around the milled areas. This deformation occurs during where the entire area was exposed to a low dose of the $\mathrm{He}^{+}$beam. Figure S2 compares the feature before and after zoomed-in $\mathrm{He}^{+}$ imaging. It is observed that the patterned features within imaged areas, corresponding to Figure $5 b-d$, are seriously deformed. This may be due to the carbon redeposition induced by imaging. Features 
in other areas remained stable. Complementary STEM images of a pattern are shown in Figure 6. Although lattice imaging was attempted in order to reveal any peripheral graphene damage, as has been observed in other two-dimensional materials [26,30,31], the residual carbon around the deposits made it impossible to directly image the lattice at an atomic resolution. Images for a corner cut are shown in Figure 6a,b and images for a vertical cut are shown in Figure $6 c, d$. The residual carbon is observed even though the samples prepared for the STEM study were preannealed before the milling (see Experimental section for details), which indicates the carbon was redeposited near the edge of the mill. Although STEM did not reveal the level of damage to the patterned area, the contrast shown in the $\mathrm{He}^{+}$microscope may suggest that the nanobridges are conductive and the lattice damage is minimal, as the previous work has shown that damage to the lattice will cause depletion of electrons to the pattern when imaging with positive ions [36].
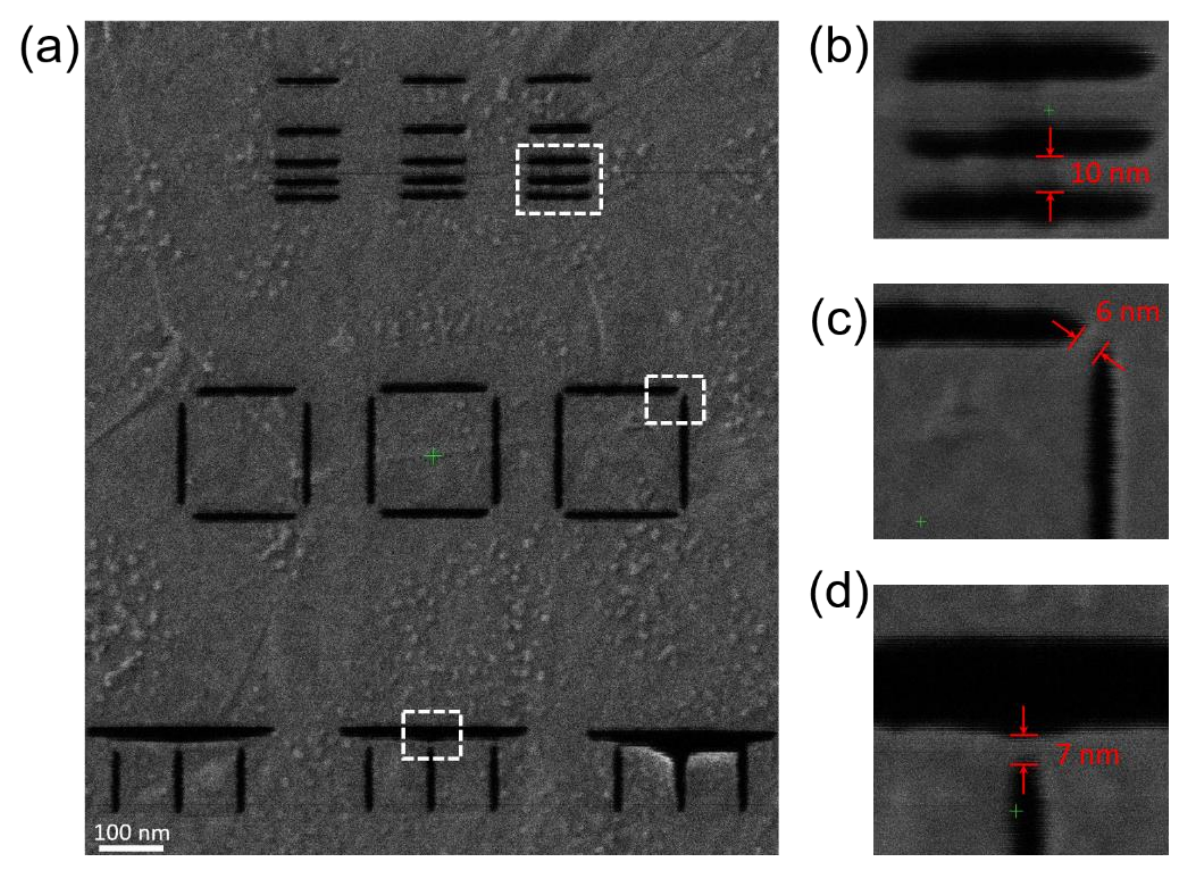

Figure 5. A series of patterns were made to explore of the highest milling resolution (a) and the patterning resolution with parallel (b), corner (c) and vertical (d) cuts. The width of the graphene nanobridges in these ions milled features can reach $10 \mathrm{~nm}$ in parallel cuts and approximately $6 \sim 7 \mathrm{~nm}$ in corner and vertical cuts. 


\section{(a)}

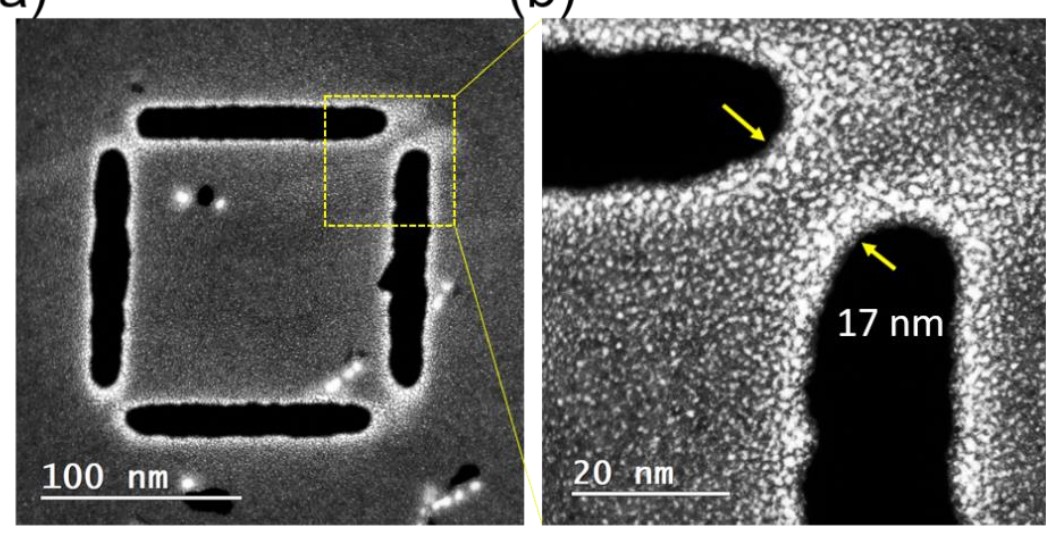

\section{(c)}

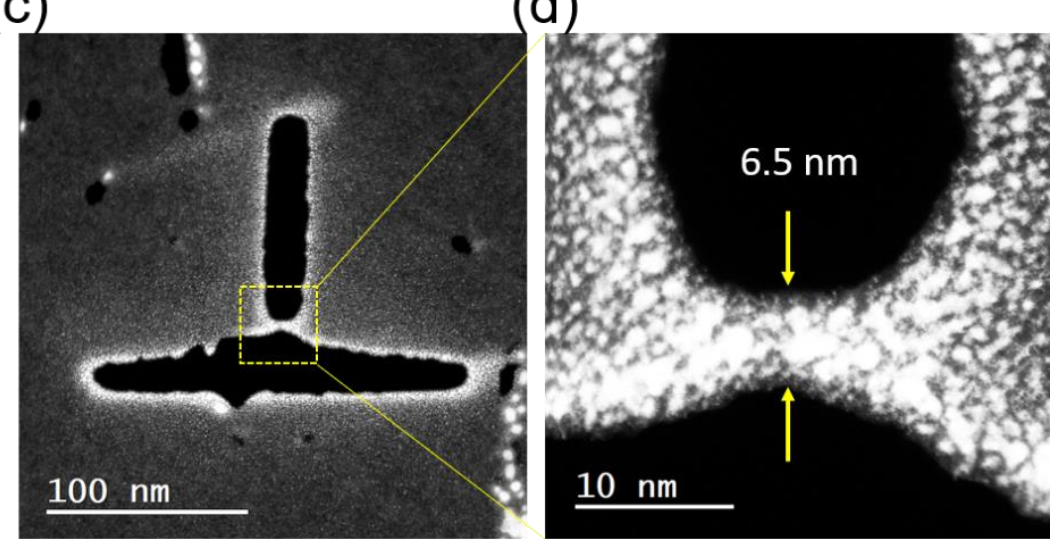

Figure 6. STEM images for preannealed graphene samples, after laser-assisted $\mathrm{He}^{+}$milling. Images of a corner cut and a vertical cut are shown in $(\mathbf{a}, \mathbf{b})$ and $(\mathbf{c}, \mathbf{d})$, respectively. Carbon redeposition is clearly observed near the milled region.

We have shown laser-assisted ion milling with sub $10 \mathrm{~nm}$ resolution, which enabled complex features to be written on these monolayer graphene samples. Figure 7 shows several examples of complex features patterned with the $48 \mathrm{~kW} / \mathrm{cm}^{2}$ irradiance $(1 \mathrm{~A}), 10 \mu$ s pulse width, and $100 \mathrm{~Hz}$ laser conditions, and a $\mathrm{He}^{+}$linear dose of $2 \times 10^{5}$ ions/nm. Figure $7 \mathrm{a}$ demonstrates a progressive milling process for a predesigned pattern, and more completed patterns are shown in Figure $7 \mathrm{~b}$. It is observed in these complex features that part of the graphene film was folded and rolled up during the milling process which resulted in the bright contrast in the images. Notably, in the last pattern shown in the right panel of Figure $7 \mathrm{~b}$, strain was intentionally introduced in the suspended nanobridges. Detailed patterning steps for this strained feature can be found in Supplementary Materials (Figure S3). These complex patterns are synthesized via a series of $\mathrm{He}^{+}$cuts similar to macro kirigami. The suspended structures can remain stable, held by the graphene bridges with widths from $20 \mathrm{~nm}$ to $50 \mathrm{~nm}$. This opens the possibility to direct write complex nanodevices on suspended membranes without traditional lithography. 
(a)

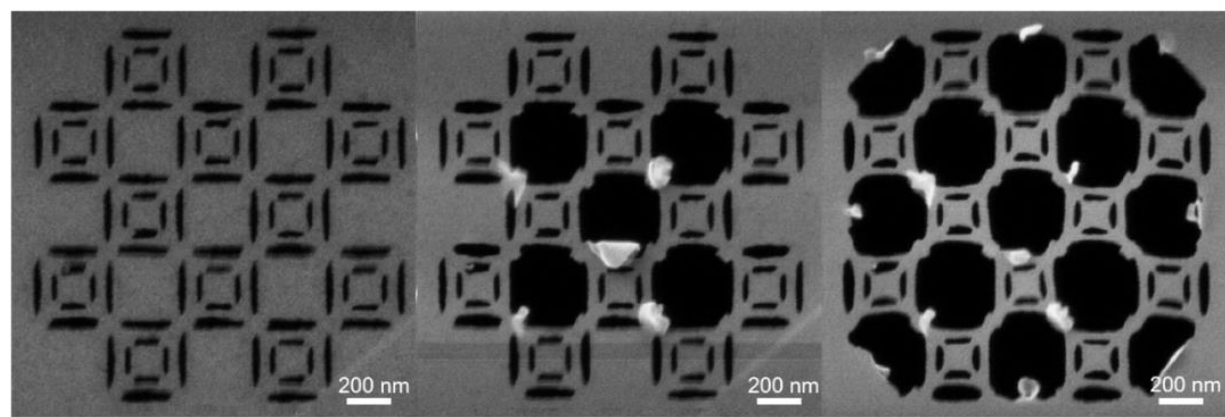

(b)

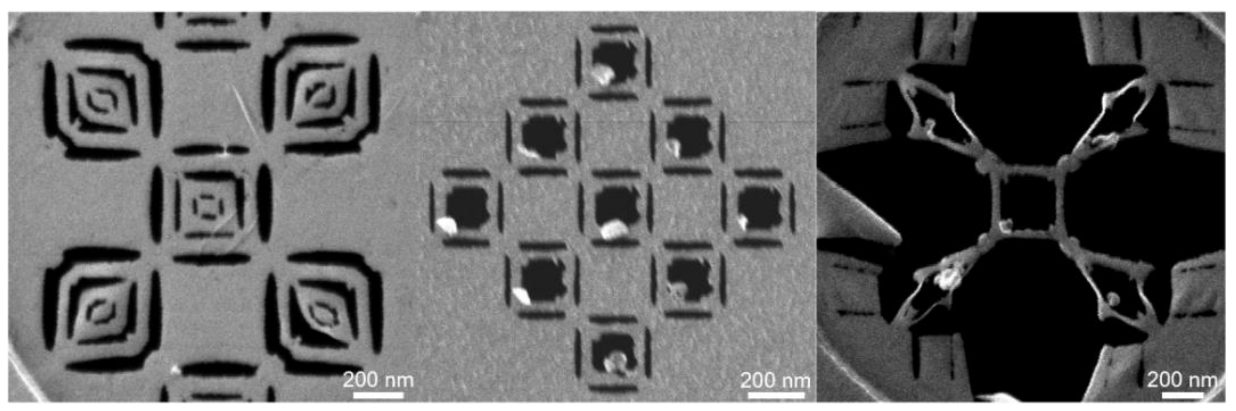

Figure 7. Complex features patterned under base parameters. (a) demonstrates a progressive milling procedure for a kirigami pattern and (b) shows several other compete patterns. With designed CAD files, these nanoscale graphene kirigami patterns can be easily realized by laser-assisted $\mathrm{He}^{+}$milling. The suspended structures are stable with these graphene bridges with the width of tens of nanometers. Note in the right panel of $(\mathbf{b})$ we intentionally induced strains in the suspended pattern and detailed steps of milling process can be found in Figure S3.

\section{Conclusions}

In summary, we have shown a systematic study on laser-assisted $\mathrm{He}^{+}$milling on monolayer graphene samples. By in situ pulsed laser, carbon contamination in commercial grade graphene samples can be effectively mitigated which enables ion milling. The detailed parameters of laser and $\mathrm{He}^{+}$beam were carefully investigated with the demonstrated patterning resolution reaching sub $10 \mathrm{~nm}$. Complex nanoscale kirigami patterns were then made with the suspended structure held by nanobridges. Heavy carbon redeposition is observed by STEM near the $\mathrm{He}^{+}$milled area This technique enables complex features to be direct written on suspended membranes, opening the gate to a variety of applications such as fabricating heterostructure carbon-based devices.

Supplementary Materials: The following are available online at http://www.mdpi.com/2079-4991/9/10/1394/s1, Figure S1: $\mathrm{He}^{+}$condition profile for graphene milling with laser; Figure S2: FEM simulation result for laser power (a) and pulse width (b) tests; Figure S3: Feature deformation due to the $\mathrm{He}^{+}$exposure; detailed description of FEM simulation.

Author Contributions: C.Z. and P.D.R. designed this study; C.Z., M.G.S., and A.A.B. conducted HIM experiments; O.D. and S.J. carried out STEM characterizations; D.A.G. and J.D.F. conducted the FEM simulation for the heating analysis; C.Z. and P.D.R. wrote the manuscript; and all authors contributed to discuss and comment on the manuscript.

Funding: The authors (P.D.R., J.D.F., A.A.B., S.J, and O.D.) acknowledge support from the Center for Nanophase Materials Sciences, which is a DOE Office of the Science User Facility. C.Z. and M.G.S. acknowledge support from the U.S. Department of Energy (DOE) under grant no. DE-SC0002136. D.A.G. acknowledges the support by NSF CBET-1603780.

Acknowledgments: We acknowledge Walker L. Boldman for the help on graphic design for the schematic figure.

Conflicts of Interest: The authors declare no conflict of interest. 


\section{References}

1. Novoselov, K.S.; Geim, A.K.; Morozov, S.V.; Jiang, D.; Zhang, Y.; Dubonos, S.V.; Grigorieva, I.V.; Firsov, A.A. Electric field effect in atomically thin carbon films. Science 2004, 306, 666-669. [CrossRef] [PubMed]

2. Chen, Z.; Lin, Y.-M.; Rooks, M.J.; Avouris, P. Graphene nano-Ribbon electronics. Phys. E Low-Dimens. Syst. Nanostructures 2007, 40, 228-232. [CrossRef]

3. Li, Z.; Liu, Z.; Sun, H.; Gao, C. Superstructured assembly of nanocarbons: Fullerenes, nanotubes, and graphene. Chem. Rev. 2015, 115, 7046-7117. [CrossRef] [PubMed]

4. Harris, P.J.F. Engineering carbon materials with electricity. Carbon 2017, 122, 504-513. [CrossRef]

5. Feng, J.; Li, W.; Qian, X.; Qi, J.; Qi, L.; Li, J. Patterning of graphene. Nanoscale 2012, 4, 4883-4899. [CrossRef] [PubMed]

6. Li, C.; Shi, G. Three-Dimensional graphene architectures. Nanoscale 2012, 4, 5549-5563. [CrossRef] [PubMed]

7. Cao, Y.; Fatemi, V.; Fang, S.; Watanabe, K.; Taniguchi, T.; Kaxiras, E.; Jarillo-Herrero, P. Unconventional superconductivity in Magic-Angle graphene superlattices. Nature 2018, 556, 43-50. [CrossRef]

8. Qi, J.S.; Huang, J.Y.; Feng, J.; Shi, D.N.; Li, J. The Possibility of Chemically Inert, Graphene-Based All-Carbon Electronic Devices with 0.8 eV Gap. ACS Nano 2011, 5, 3475-3482. [CrossRef] [PubMed]

9. Yi, C.; Chen, X.; Zhang, L.; Wang, X.; Ke, C. Nanomechanical Z-Shape folding of graphene on flat substrate. Extrem. Mech. Lett. 2016, 9, 84-90. [CrossRef]

10. Mu, J.; Hou, C.; Wang, H.; Li, Y.; Zhang, Q.; Zhu, M. Origami-Inspired active Graphene-Based paper for programmable instant Self-Folding walking devices. Sci. Adv. 2015, 1, e1500533. [CrossRef]

11. Zhu, S.; Li, T. Hydrogenation-Assisted Graphene Origami and Its Application in Programmable Molecular Mass Uptake, Storage, and Release. ACS Nano 2014, 8, 2864-2872. [CrossRef] [PubMed]

12. Grosso, B.F.; Mele, E.J. Bending Rules in Graphene Kirigami. Phys. Rev. Lett. 2015, 115, 195501. [CrossRef] [PubMed]

13. Qi, Z.; Campbell, D.K.; Park, H.S. Atomistic simulations of Tension-Induced large deformation and stretchability in graphene kirigami. Phys. Rev. B 2014, 90, 245437. [CrossRef]

14. Park, O.-K.; Tiwary, C.S.; Yang, Y.; Bhowmick, S.; Vinod, S.; Zhang, Q.; Colvin, V.L.; Asif, S.A.S.; Vajtai, R.; Penev, E.S.; et al. Magnetic field controlled graphene oxide-based origami with enhanced surface area and mechanical properties. Nanoscale 2017, 9, 6991-6997. [CrossRef] [PubMed]

15. Ebbesen, T.W.; Hiura, H. Graphene in 3-Dimensions: Towards graphite origami. Adv. Mater. 1995, 7, 582-586. [CrossRef]

16. Blees, M.K.; Barnard, A.W.; Rose, P.A.; Roberts, S.P.; McGill, K.L.; Huang, P.Y.; Ruyack, A.R.; Kevek, J.W.; Kobrin, B.; Muller, D.A.; et al. Graphene kirigami. Nature 2015, 524, 204-207. [CrossRef] [PubMed]

17. Saadani, A.; Lavenus, P.; Traon, O.L.; Héripré, E.; Sthal, F. FIB-Induced damage in graphene electrodes for piezoelectric resonators. In Proceedings of the 2018 Symposium on Design, Test, Integration \& Packaging of MEMS and MOEMS (DTIP), Roma, Italy, 22-25 May 2018; pp. 1-3.

18. Kim, S.; Dyck, O.; Ievlev, A.V.; Vlassiouk, I.V.; Kalinin, S.V.; Belianinov, A.; Jesse, S.; Ovchinnikova, O.S. Graphene milling dynamics during helium ion beam irradiation. Carbon 2018, 138, 277-282. [CrossRef]

19. Lemme, M.C.; Bell, D.C.; Williams, J.R.; Stern, L.A.; Baugher, B.W.H.; Jarillo-Herrero, P.; Marcus, C.M. Etching of Graphene Devices with a Helium Ion Beam. ACS Nano 2009, 3, 2674-2676. [CrossRef] [PubMed]

20. Miller, D.; Blaikie, A.; Carter, B.; Alemán, B. Engineering the Modal Shape of Graphene Nanoelectromechanical Systems Using Focused Ion Beam Milling. In Proceedings of the 2018 IEEE 13th Nanotechnology Materials and Devices Conference (NMDC), Portland, OR, USA, 14-17 October 2018; pp. 1-4.

21. Schmidt, M.E.; Iwasaki, T.; Muruganathan, M.; Haque, M.; Van Ngoc, H.; Ogawa, S.; Mizuta, H. Structurally Controlled Large-Area $10 \mathrm{~nm}$ Pitch Graphene Nanomesh by Focused Helium Ion Beam Milling. Acs Appl. Mater. Inter. 2018, 10, 10362-10368. [CrossRef]

22. Araujo, E.N.D.; Brant, J.C.; Archanjo, B.S.; Medeiros-Ribeiro, G.; Alves, E.S. Quantum corrections to conductivity in graphene with vacancies. Phys. E: Low-Dimens. Syst. Nanostructures 2018, 100, 40-44. [CrossRef]

23. Dyck, O.; Kim, S.; Kalinin, S.V.; Jesse, S. Graphene Defect Editing, Deposition, and Growth via E-Beam-Induced Organic Reactions in Aberration Corrected STEM. Microsc. Microanal. 2018, 24, 1994-1995. [CrossRef] 
24. Kim, S.; Ievlev, A.V.; Jakowski, J.; Vlassiouk, I.V.; Sang, X.; Brown, C.; Dyck, O.; Unocic, R.R.; Kalinin, S.V.; Belianinov, A.; et al. Multi-Purposed Ar gas cluster ion beam processing for graphene engineering. Carbon 2018, 131, 142-148. [CrossRef]

25. Stanford, M.G.; Lewis, B.B.; Noh, J.H.; Fowlkes, J.D.; Roberts, N.A.; Plank, H.; Rack, P.D. Purification of Nanoscale Electron-Beam-Induced Platinum Deposits via a Pulsed Laser-Induced Oxidation Reaction. Acs Appl. Mater. Inter. 2014, 6, 21256-21263. [CrossRef] [PubMed]

26. Stanford, M.G.; Pudasaini, P.R.; Belianinov, A.; Cross, N.; Noh, J.H.; Koehler, M.R.; Mandrus, D.G.; Duscher, G.; Rondinone, A.J.; Ivanov, I.N.; et al. Focused Helium-Ion beam irradiation effects on electrical transport properties of Few-Layer WSe2: Enabling nanoscale direct write Homo-Junctions. Sci. Rep. 2016, 6, 27276. [CrossRef]

27. Lewis, B.B.; Winkler, R.; Sang, X.; Pudasaini, P.R.; Stanford, M.G.; Plank, H.; Unocic, R.R.; Fowlkes, J.D.; Rack, P.D. 3D Nanoprinting via Laser-Assisted electron beam induced deposition: Growth kinetics, enhanced purity, and electrical resistivity. Beilstein J. Nanotechnol. 2017, 8, 801-812. [CrossRef]

28. Stanford, M.G.; Lewis, B.B.; Noh, J.H.; Fowlkes, J.D.; Rack, P.D. Inert Gas Enhanced Laser-Assisted Purification of Platinum Electron-Beam-Induced Deposits. Acs Appl. Mater. Inter. 2015, 7, 19579-19588. [CrossRef] [PubMed]

29. Stanford, M.G.; Lewis, B.B.; Iberi, V.; Fowlkes, J.D.; Tan, S.; Livengood, R.; Rack, P.D. In Situ Mitigation of Subsurface and Peripheral Focused Ion Beam Damage via Simultaneous Pulsed Laser Heating. Small 2016, 12, 1779-1787. [CrossRef]

30. Noh, J.H.; Fowlkes, J.D.; Timilsina, R.; Stanford, M.G.; Lewis, B.B.; Rack, P.D. Pulsed Laser-Assisted Focused Electron-Beam-Induced Etching of Titanium with XeF2: Enhanced Reaction Rate and Precursor Transport. Acs Appl. Mater. Inter. 2015, 7, 4179-4184. [CrossRef] [PubMed]

31. Stanford, M.G.; Mahady, K.; Lewis, B.B.; Fowlkes, J.D.; Tan, S.; Livengood, R.; Magel, G.A.; Moore, T.M.; Rack, P.D. Laser-Assisted Focused He+ Ion Beam Induced Etching with and without XeF2 Gas Assist. Acs Appl. Mater. Inter. 2016, 8, 29155-29162. [CrossRef]

32. Dyck, O.; Ziatdinov, M.; Lingerfelt, D.B.; Unocic, R.R.; Hudak, B.M.; Lupini, A.R.; Jesse, S.; Kalinin, S.V. Atom-By-Atom fabrication with electron beams. Nat. Rev. Mater. 2019, 4, 497-507. [CrossRef]

33. Susi, T.; Meyer, J.C.; Kotakoski, J. Quantifying transmission electron microscopy irradiation effects using two-dimensional materials. Nat. Rev. Phys. 2019, 1, 397-405. [CrossRef]

34. Dyck, O.; Kim, S.; Kalinin, S.V.; Jesse, S. Mitigating E-Beam-Induced hydrocarbon deposition on graphene for Atomic-Scale scanning transmission electron microscopy studies. J. Vac. Sci. Technol. B 2018, 36, 011801. [CrossRef]

35. Do, D.D.; Do, H.D. Surface Diffusion of Hydrocarbons in Activated Carbon: Comparison Between Constant Molar Flow, Differential Permeation and Differential Adsorption Bed Methods. Adsorption 2001, 7, 189-209. [CrossRef]

36. Iberi, V.; Vlassiouk, I.; Zhang, X.G.; Matola, B.; Linn, A.; Joy, D.C.; Rondinone, A.J. Maskless Lithography and in situ Visualization of Conductivity of Graphene using Helium Ion Microscopy. Sci. Rep. 2015, 5, 11952. [CrossRef] [PubMed]

(C) 2019 by the authors. Licensee MDPI, Basel, Switzerland. This article is an open access article distributed under the terms and conditions of the Creative Commons Attribution (CC BY) license (http://creativecommons.org/licenses/by/4.0/). 